\title{
Desacuerdo lógico y agregación ${ }^{*}$
}

\author{
(Logical Disagreement and Aggregation)
}

\author{
Diego TAJER
}

Received: 29/01/22016

Final Version: 27/08/2016

BIBLID 0495-4548(2017)32:1p.63-87

DOI: $10.1387 /$ theoria. 15716

RESUMEN: En este artículo, exploro la posibilidad de aplicar los métodos y resultados de la Agregación de Juicios al problema del desacuerdo lógico. Es decir, desarrollo y evalúo maneras en que individuos que desacuerdan lógicamente pueden generar una lógica colectiva. Pruebo una versión de la paradoja discursiva, donde el voto por mayoría en un grupo de lógicos estructurales puede generar lógicas subestructurales, y luego elaboro un resultado de imposibilidad más general. Más adelante, analizo diferentes maneras de evitar la paradoja, en particular aquellas que apelan a restricciones de dominio. Finalmente, analizo la aplicación de estos resultados al debate sobre paradojas semánticas.

Palabras clave: Desacuerdo lógico; Agregación de juicios; Lógicas no-clásicas; Paradojas semánticas.

ABSTRACT: In this paper, I explore the possibility of applying the methods and results of Judgement Aggregation to the problem of logical disagreement. I develop and evaluate different ways in which individuals who logically disagree can generate a collective logic. I prove a version of the discursive paradox, where the majority voting of a group of structural logicians can give rise to a substructural logic; then I develop a more general impossibility result. After this, I analyze different ways to avoid the paradox, in particular some methods which appeal to domain restrictions. Finally, I consider the application of these results to the discussion on semantic paradoxes.

Keywords: Logical disagreement; Judgement Aggregation; Non-classical logics; Semantic paradoxes.

\section{Introducción}

Es común en filosofía de la lógica admitir que distintos agentes pueden sostener distintas lógicas racionalmente. Estos desacuerdos, en ocasiones, también necesitan de la generación de consensos.

Supongamos que un determinado argumento se le presenta a un grupo de individuos que aceptan distintas lógicas. Si el argumento en cuestión no es válido en todas las lógicas que aceptan los miembros del grupo, se producirá algún tipo de discusión. Los agentes tienen, naturalmente, la opción de refugiarse en sus propias opiniones. Pero también pueden intentar consensuar una respuesta grupal al problema.

La situación no es cualitativamente distinta al caso de la agregación de juicios (List, 2012), donde un grupo de personas intenta determinar la verdad o falsedad de un

* Agradezco a los miembros de Buenos Aires Logic Group, y a los participantes del IV Workshop on Philosophical Logic (Buenos Aires, 2015), por ayudarme a elaborar las ideas de este artículo. También agradezco a dos réferis anónimos por los detallados y útiles comentarios. 
conjunto de enunciados. Probablemente tendrán distintas opiniones, pero eso no significa que renuncien a una actitud constructiva, de búsqueda de consenso grupal. En esta dirección, podemos explorar si los métodos de agregación de juicios pueden utilizarse también como métodos para agregación de lógicas, para entender cómo individuos que desacuerdan lógicamente pueden alcanzar posiciones colectivas.

Los métodos de agregación o consenso grupal en lógica a veces se presuponen en ciertas discusiones. Por ejemplo, frecuentemente se argumenta de este modo: «no podemos rechazar $I$, porque es una inferencia que casi todos aceptan como válida» ${ }^{1}$. Ese tipo de argumentaciones apelan a cierta comunidad, donde la mayoría (o una super-mayoría) acepta determinada inferencia, incluso cuando esa mayoría puede no estar de acuerdo con respecto a otras reglas o principios. La cantidad de individuos expertos que aceptan ciertas inferencias (o nuestra estimación aproximada de esa cantidad) es un factor que puede influir sobre nuestro apoyo o rechazo de esas inferencias. En ocasiones puede no aparecer una apelación explícita a la cantidad de personas que aceptan una regla, sino al rol central de esa regla en la práctica científica. Y claramente, una regla no podría tener un rol central en la comunidad si no fuera apoyada por la mayor parte de los miembros.

Asimismo, hay muchos casos donde los métodos de agregación de lógicas podrían usarse explícitamente. Muchas veces, distintos individuos (o grupos) aceptan distintos principios lógicos respecto a un problema específico. Esto sucede con diversas paradojas, aunque el ejemplo más claro es la paradoja del Mentiroso. En esa discusión, algunos agentes (los clásicos) se inclinan por rechazar la intersustitutividad entre $\varphi$ y $T(\langle\varphi\rangle)$, otros (los paracompletos) prefieren rechazar el principio del tercero excluido, otros (los paraconsistentes) rechazan Explosión, etc. Es difícil encontrar razones excluyentes a favor o en contra de alguna de esas soluciones. Dado ese escenario, si uno propone la solución $S$, uno puede considerar como un par epistémico a quien propone la solución distinta $S$ '. Es decir, el que sostiene la posición distinta es «similar respecto a la familiaridad con la evidencia y con los argumentos sobre el asunto» (Kelly 2005, p. 174). Tener en cuenta la opinión de un par, cuando no hay certeza en la propia posición, es una actitud racional. El argumento puede generalizarse para el caso grupal: si un determinado grupo propone distintas soluciones a un mismo problema, y todas esas soluciones se encuentran en igualdad de condiciones, podemos decir que es un desacuerdo entre pares epistémicos. Entonces, es razonable buscar una solución grupal, en donde los puntos de vista de todos los agentes del grupo sean tenidos en cuenta.

Como es de esperar, hay muchos mecanismos para construir consensos grupales. Algunos serán mejores que otros, según los objetivos que estemos buscando. Pero no hay razones de principio para descartar la formación de lógicas «colectivas», a menos que estemos dispuestos a cuestionar el estudio de mecanismos de agregación en general ${ }^{2}$.

1 Si bien esta posición nunca fue defendida explícitamente, está implícitamente contenida en Williamson (2013). Aquí se sostiene que la lógica clásica, al ser sostenida por la mayor parte de la comunidad, puede ser utilizada como punto de referencia para entender las otras lógicas. De modo que revisar la lógica clásica es injustificado, a menos que uno encuentre otra lógica que tenga en términos globales menos defectos que la clásica, y pueda cumplir esa condición de punto de referencia. No basta con encontrar un argumento específico para revisar los principios clásicos.

2 Si bien la agregación de lógicas nunca fue abordada, los mecanismos de agregación de juicios fueron aplicados para otras construcciones abstractas, en particular para argumentos. Esta línea fue adoptada por Rahwan y Tohme (2010). 


\section{Agregación de juicios}

Los métodos de agregación de lógicas que voy a investigar se basan en la teoría de agregación de juicios. Esta teoría estudia mecanismos por los cuales un grupo compuesto de individuos con juicios distintos puede formar juicios colectivos (o grupales) sobre determinado asunto. Los agentes deliberan sobre un conjunto determinado de proposiciones. En términos formales, una agenda $X$ es un conjunto de oraciones de un lenguaje proposicional $L$ que representa a las proposiciones sobre las cuales el grupo vota. La agenda está cerrada bajo negación (si $\varphi \in X$, entonces $\neg \varphi \in X)$. Siguiendo a List (2012, fn 5), en algunos casos haré una simplificación para evitar que las agendas sean necesariamente infinitas. La simplificación es que las dobles negaciones se «cancelan» (i.e. no hace falta incluir $\neg \neg \varphi$ cuando $\varphi$ ya está en la agenda): entonces $\{p, \neg p\}$ puede ser una agenda, por ejemplo. En ocasiones, usaremos la expresión « $₫ \Gamma \gg$ para definir a una agenda, donde $\pm \Gamma=\Gamma \cup\{\neg \gamma \mid \gamma \in \Gamma\}$.

Un conjunto de aceptación $A$ sobre una agenda $X$ es un subconjunto de oraciones de $X$, que es completo relativamente a la agenda (es decir, para todo $\operatorname{par}\{\varphi, \neg \varphi\} \in X, \varphi \in A$ o $\neg \varphi \in A$ ) y consistente. El conjunto de aceptación puede representar lo que un agente acepta (es decir, $A_{i}$ ), o también lo que acepta un grupo ${ }^{3}$.

Un perfil $P$ es una $n$-tupla $\left\langle A_{1}, \ldots, A_{n}\right\rangle$ de conjuntos de aceptación, con un lugar para cada individuo del grupo $G$. Es decir, el perfil representará, para un conjunto de agentes, lo que cada uno de esos agentes acepta en un determinado momento. Por ejemplo, supongamos que la agenda es $X= \pm\{p, q\}$ y el grupo es $G=\{1,2,3\}$. Un conjunto de aceptación podría ser $A_{1}=\{p, \neg q\}$. Este conjunto es completo respecto a la agenda, y representaría los juicios del agente 1 . Por otro lado, un perfil podría ser el siguiente:

$$
<A_{1}, A_{2}, A_{3}>=<\{p, \neg q\},\{p, q\},\{p, \neg q\}>
$$

Es decir, un perfil $P$ representa los compromisos de un conjunto determinado de agentes, en este caso los agentes 1,2 y 3 .

Sea $\boldsymbol{P}^{*}$ el conjunto de todos los perfiles posibles, y $\boldsymbol{P}$ un conjunto de perfiles. Un mecanismo de agregación es una función que toma perfiles $P \in \boldsymbol{P}$ y arroja subconjuntos de $X^{4}$ :

$$
f: \quad \boldsymbol{P}^{*} \supseteq \boldsymbol{P} \quad \rightarrow \quad \wp(X)
$$

Es decir, un mecanismo de agregación es una función que toma las opiniones de los individuos de un grupo, y arroja un conjunto de oraciones único (el «conjunto colectivo»), que viene a representar el conjunto de juicios grupales.

Sobre las funciones pueden imponerse diversas condiciones.

(Dominio irrestricto) $\quad \mathbf{P}=\mathbf{P}^{*}$ (i.e. cualquier perfil es admisible)

(Racionalidad colectiva) $f\left(A_{1}, \ldots, A_{n}\right)$ es un conjunto de aceptación (i.e. la función de agregación arroja un conjunto consistente y completo respecto a la agenda)

3 Aquí tomo la aceptación, el juicio y la creencia como equivalentes. Las posibles diferencias entre estos conceptos no juegan ningún rol en esta discusión.

4 En ocasiones se asume que el output de la función debe ser un conjunto de aceptación, es decir, un conjunto consistente y completo. En este contexto, donde nos enfocamos en los resultados de imposibilidad, relajamos ese requisito. 
(Unanimidad) Si $\varphi \in \mathrm{A}_{i}$ para todo $i$, entonces $\varphi \in f\left(A_{1}, \ldots, A_{n}\right)$ (i.e. si todos los agentes aceptan una proposición, el grupo también la acepta)

(Sistematicidad) Si para todo $i, \varphi \in A_{i}$ sii $\psi \in A_{i}^{\prime}$, entonces $\varphi \in f\left(A_{1}, \ldots, A_{n}\right)$ sii $\psi \in f\left(A_{1}^{\prime}, \ldots, A_{n}^{\prime}\right)$ (i.e. si todos los individuos en dos perfiles distintos concuerdan en el patrón de aceptación de dos fórmulas, el juicio colectivo resultante para esas fórmulas también es el mismo en ambos perfiles) $)^{5}$.

Los mecanismos de agregación $f$ pueden basarse en distintos criterios. Algunos de los mecanismos más conocidos son:

- Regla de la mayoría: $\varphi$ pertenece al conjunto colectivo sii el número de personas que acepta $\varphi$ es mayor al número de personas que no acepta $\varphi$. En términos formales (cuando $\ll|\Gamma| \gg$ indica la cardinalidad de $\Gamma$ ), $f$ es un mecanismo por mayoría cuando para todo perfil $\left(A_{1}, \ldots, A_{n}\right)$ :

$$
\varphi \in f\left(A_{1}, \ldots, A_{n}\right) \operatorname{sii}\left|\left\{n \in G \mid \varphi \in A_{n}\right\}\right|>\left|\left\{n \in G \mid \varphi \notin A_{n}\right\}\right| .
$$

- Dictadura: hay un individuo $a$ tal que, en todo perfil, el grupo acepta $\varphi$ sii $a$ acepta $\varphi$. Formalmente, $f$ es un mecanismo dictatorial cuando: existe un agente $k$ tal que para cualquier perfil $\left(A_{1}, \ldots, A_{n}\right), f\left(A_{l}, \ldots, A_{n}\right)=A_{k}$.

- Regla de unanimidad: el grupo acepta $\varphi$ sii todos los individuos del grupo aceptan $\varphi$. En términos formales, $f$ es un mecanismo de unanimidad cuando para todo perfil $\left(A_{1}, \ldots, A_{n}\right)$ :

$$
\varphi \in f\left(A_{1}, \ldots, A_{n}\right) \text { sii } \varphi \in A_{i} \text { para todo } i
$$

Para ilustrar estos mecanismos, pensemos en el siguiente perfil:

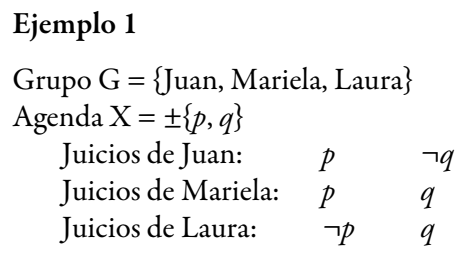

Si usamos el mecanismo de unanimidad, el grupo G no aceptará ninguna de las proposiciones de la agenda, porque no hay acuerdo unánime sobre ninguna de ellas. Si usamos la regla de mayoría, el grupo G aceptará $p$ y $q$ (porque una mayoría del grupo acepta $p$, y otra mayoría del grupo acepta $q$ ). Si usamos la regla de dictadura, sólo es cuestión de elegir un dictador y el grupo aceptará las mismas proposiciones que éste.

5 Esta explicación de Sistematicidad está en Pigozzi (2014, p. 29). En List \& Pettit (2004, p. 215) aparece una descripción algo más compleja de esta propiedad. Para cada perfil $A=\left(A_{1}, \ldots, A_{n}\right)$ y cada proposición $\varphi$, podemos definir el patrón $A_{\varphi}$ como el vector $V_{\mathrm{A}, \varphi}=\left(v_{1}, \ldots, v_{n}\right)$, donde $v_{i}=1 \operatorname{sii} \varphi \in A_{i}, \mathrm{y} v_{i}=0$ sii $\varphi \notin A_{i}$. Sistematicidad nos dice que existe una función $f$, que va de vectores $\{0,1\}^{n}$ al conjunto $\{0,1\}$, tal que el conjunto colectivo de cualquier perfil $A$ incluye una proposición $\varphi$ si y sólo si $f\left(V_{A, \varphi}\right)=1$. En este sentido, el criterio para aceptar colectivamente cualquier proposición es el mismo en todo perfil (sólo depende de la función $f$ ). 
Algunos de estos mecanismos pueden dar lugar a paradojas. La más famosa de ellas es la paradoja discursiva (Pettit 2001), que muestra que, si el dominio de $f$ es irrestricto y la agenda incluye proposiciones como $\{p, q,(p \wedge q)\}$, la regla de la mayoría no garantiza la consistencia del conjunto colectivo.

Considérese el siguiente perfil:

Ejemplo 2 (Paradoja discursiva)

\begin{tabular}{llll} 
Agenda $X=$ & \multicolumn{4}{l}{$\pm\{p, q,(p \wedge q)\}$} \\
Grupo $G=$ & \multicolumn{4}{l}{ Juan, Mariela, Laura } \\
Juicios de Juan: & $p$ & $\neg q$ & $\neg(p \wedge q)$ \\
Juicios de Mariela: & $\neg p$ & $q$ & $\neg(p \wedge q)$ \\
Juicios de Laura: & $p$ & $q$ & $p \wedge q$ \\
Mayoría: & $p$ & $q$ & $\neg(p \wedge q)$
\end{tabular}

Si aplicamos el voto por mayoría, el conjunto colectivo será inconsistente: incluirá $p, q$ y $\neg(p \wedge q)$. Porque una mayoría acepta $p$, otra mayoría acepta $q$, y otra mayoría acepta $\neg(p \wedge q)$. De este modo, un grupo de individuos con juicios consistentes genera un conjunto colectivo inconsistente.

\section{Soluciones tradicionales}

Ante la paradoja discursiva hay distintas reacciones posibles. La primera es renunciar a Racionalidad Colectiva, y pensar cómo un conjunto inconsistente de juicios puede ser racional para un grupo. Hay dos maneras de argumentar en este sentido. Por un lado, podemos decir que los grupos no son agentes colectivos, y por ende no es necesario atribuirles una racionalidad lógica tan estricta. Esta reacción es obviamente la más extrema, porque toma un fenómeno intuitivamente problemático (la inconsistencia del conjunto colectivo) e intenta transformarlo en algo esperable o razonable. Briggs et al. (2014) y List (2015) proponen una respuesta más elaborada. Ellos sostienen que en ocasiones un grupo no requiere de consistencia, sino de 2-consistencia, es decir, que todos los pares de oraciones adoptadas por el grupo sean consistentes (aunque no necesariamente los conjuntos de más de 2 elementos). Esta idea puede verse como una adaptación de la paradoja del prefacio (Makinson 1965): así como permitimos (en la paradoja del prefacio) que un individuo tenga creencias del tipo $\left\{p_{1}, \ldots, p_{n}, \neg\left(p_{1} \wedge \ldots \wedge p_{n}\right)\right\}$, podríamos permitir que un grupo tenga juicios del tipo $\{p, q, \neg(p \wedge q)\}$. De hecho, hay una correspondencia entre la $n$-consistencia y la respuesta probabilística a la paradoja del prefacio: un conjunto de individuos que votan por mayoría siempre obtendrán un conjunto 2-consistente, cuyas oraciones pueden tener probabilidad $>.5$ bajo una asignación probabilística (véase List 2015). En el caso recién mencionado, el conjunto colectivo es $\{p, q, \neg(p \wedge q)\}$, que obviamente es 2-consistente.

Una segunda solución posible es usar la regla de la mayoría para las «premisas», en este caso $p$ y $q$. La regla de premisas funciona de la siguiente manera:

- Regla de (mayoría para) premisas: Para un subconjunto $Y$ («premisas») de la agenda $X, \varphi \in Y$ pertenece al conjunto colectivo sii el número de personas que acepta $\varphi$ es mayor al número de personas que no acepta $\varphi$. El resto del conjunto co- 
lectivo se determina a partir de las consecuencias de estas oraciones. En términos formales, $f$ es una regla de premisas cuando, siendo $h$ una regla de mayoría, y siendo $Y \subset X$, sucede lo siguiente en todo perfil $\left(A_{l}, \ldots, A_{n}\right)$ :

$$
\varphi \in f\left(A_{1}, \ldots, A_{n}\right) \operatorname{sii} h\left(A_{1}, \ldots, A_{n}\right) \cap Y \vDash \varphi
$$

Lo importante para evitar inconsistencias colectivas es que las premisas sean lógicamente independientes (es decir, que para cualesquiera dos oraciones $\varphi$ y $\psi \in Y, \varphi \not \models \psi$ y $\psi \not \models \varphi$ ). Si las premisas también son una base lógica de la agenda (es decir, si a partir de cualquier conjunto completo respecto a $Y$ podemos probar $\varphi$ o $\neg \varphi$ para cada $\varphi \in X$ ), entonces la regla de premisas siempre arrojará un conjunto de aceptación completo (cuando el número de agentes es impar). De este modo, para el caso de la paradoja discursiva, y tomando $p$ y $q$ como las premisas, el grupo $\mathrm{G}$ creería $p$ y $q$, y por ende también $p \wedge q$. Esta es la solución preferida por Pettit (2001), por ejemplo, porque permite adoptar grupalmente una proposición siempre que la mayoría haya aceptado las «razones» para esa proposición.

Sin embargo, la regla de premisas tiene dos claros problemas. En primer lugar, a veces no resulta tan claro qué oraciones son premisas, y decidir sobre el asunto genera fuertes divergencias en el resultado final (véase Pigozzi 2006: 288). En segundo lugar, la regla de premisas habilita estrategias de manipulación, donde visto el resultado de votar su creencia honesta, un agente adopta una opinión no-honesta para hacer que el voto grupal sobre otra proposición arroje un resultado conveniente. Por ejemplo, en el ejemplo 2 , Mariela cree $\{\neg p, q, \neg(p \wedge q)\}$. Pero sabe que se vota por premisas y que los votos de los otros implican la imposibilidad de ese resultado. Suponiendo que a Mariela le interesara particularmente que el grupo decida $\neg(p \wedge q)$, ella puede lograr que el grupo vote esa proposición. Para eso, puede falsear su opinión sobre $q$ y votar el siguiente conjunto $\{\neg p, \neg q, \neg(p \wedge q)\}$. Si esto sucede, siguiendo el voto por premisas el grupo ahora aceptará $\neg(p \wedge q)$, aún cuando los votos individuales sobre esta proposición sean iguales a los del perfil anterior.

\section{Restricción de agenda}

Una manera distinta de resolver el dilema discursivo es restringir la agenda, es decir, el conjunto de proposiciones sobre las cuales las personas votan. El problema aquí es que las restricciones deben ser muy extremas: si tenemos $p, q$, y alguna de las fórmulas que conectan $p$ y $q$ con un conectivo veritativo-funcional ( $\operatorname{como} p \wedge q, p \vee q$ o $p \rightarrow q$ ) en la agenda, ya se producirá el escenario paradójico (este era el resultado original de List y Pettit 2002).

Más aún, hay maneras precisas de determinar qué agendas se ven afectadas por la paradoja discursiva. Aquí entra en juego el concepto de una agenda «mínimamente conectada»:

Definición 3. Decimos que un conjunto $X$ es minimamente inconsistente sii $X$ es inconsistente, pero todo subconjunto $Y \subset X$ es consistente.

Definición 4. Decimos que una agenda está minimamente conectada sii incluye un subconjunto $Y$ mínimamente inconsistente de cardinalidad mayor o igual a 3; e incluye un subconjunto $\mathrm{Y}^{*}$ mínimamente inconsistente tal que $\left(Y^{*}-Z\right) \cup\{\neg z \mid z \in Z\}$ es consistente, para un conjunto $Z$ de cardinalidad par. 
Por ejemplo, la agenda $\pm\{p, q, p \wedge q\}$ está mínimamente conectada. Porque incluye el subconjunto $\{p, q, \neg(p \wedge q)\}$, que es mínimamente inconsistente y de cardinalidad 3; y asimismo, $(\{p, q, \neg(p \wedge q)\}-\{p, q\}) \cup\{\neg p, \neg q\}=\{\neg p, \neg q, \neg(p \wedge q)\}$, es consistente.

Si uno aplica la estrategia de restricción de agenda, lo importante es evitar que las agendas estén mínimamente conectadas. Pues, si la agenda está mínimamente conectada, el voto por mayoría no garantiza que el resultado sea consistente y completo. En otras palabras, podemos usar el siguiente resultado:

Teorema 5. Si una agenda está mínimamente conectada y el dominio es irrestricto, una función de agregación que cumple Unanimidad, Sistematicidad y Racionalidad Colectiva es una dictadura.

Prueba. Véase Dietrich y List (2007) o Pigozzi (2014: 44).

De aquí podemos derivar que si el dominio es irrestricto y la agenda está mínimamente conectada, el voto por mayoría no cumple con Racionalidad Colectiva. Pues el voto por mayoría cumple con Unanimidad y Sistematicidad, y no es un mecanismo dictatorial ${ }^{6}$. Cuando el número de agentes es impar, la falla de Racionalidad Colectiva significa que el voto por mayoría puede arrojar un resultado inconsistente.

En general, no es fácil dar restricciones razonables que dejen afuera a las agendas mínimamente conectadas, más allá del criterio extremo de reducir las agendas a letras proposicionales y sus negaciones (evitando así cualquier tipo de conexión entre las proposiciones de la agenda). Por eso la restricción de la agenda es una estrategia poco prometedora.

\section{Restricción de dominio}

List y Pettit (2002) mostraron otra salida para la paradoja discursiva: rechazar Dominio Irrestricto, y postular alguna restricción sobre el dominio, es decir, sobre el conjunto de perfiles admitidos. La restricción que proponen estos autores es la alineación uniforme:

(Alineación Uniforme). Un perfil está uniformemente alineado sii los individuos pueden ser ordenados linealmente de izquierda a derecha de modo que, para cada proposición $\varphi$, los individuos que la aceptan están todos a la derecha o todos a la izquierda, respecto a los individuos que la rechazan.

En ocasiones se dice que la alineación uniforme puede corresponder con una alineación «ideológica» de derecha a izquierda de los votantes (List 2012). Para cada asunto «izquierdista» (supongamos, la legalidad del aborto en el tercer mes), si alguien de centro lo acepta, entonces todos a su izquierda lo aceptarán. Para cada asunto «derechista» (supongamos, la prohibición del casamiento homosexual), si alguien de centro lo acepta, entonces todos a su derecha lo aceptarán.

6 El mecanismo de la mayoría cumple Unanimidad porque obviamente el grupo entero es una mayoría. Cumple Sistematicidad porque si un grupo decide sobre una proposición, decide sobre cualquier otra proposición con el mismo patrón de aceptación en cualquier otro perfil, porque se trata de una mayoría. Por último, es obviamente un mecanismo no-dictatorial (suponiendo que hay 3 o más agentes): ningún individuo $i$ puede ser dictador, porque si $i$ acepta p mientras todos salvo él aceptan $\neg p$, el conjunto colectivo incluirá $\neg p$. 
A partir de este concepto es posible probar el siguiente teorema:

Teorema 6 (List y Pettit 2002: 102). Si el dominio incluye solamente perfiles uniformemente alineados y el número de votantes es impar, entonces el voto por mayoría cumple con Racionalidad Colectiva.

Prueba. En todo perfil de ese dominio, el voto por mayoría corresponderá a lo que dice el votante medio, es decir, aquel que está en la mitad de la tabla. Si el votante medio acepta el enunciado $\varphi$, entonces todos a su derecha, o todos a su izquierda, lo aceptan. Por ende, la mayoría lo acepta. Si el votante medio rechaza $\varphi$, entonces todos a su derecha, o todos a su izquierda, lo rechazan. Por ende, la mayoría lo rechaza. Claramente, esto no constituye una dictadura, porque el votante medio puede ser un agente distinto en distintos perfiles.

Más adelante exploraremos la posibilidad de aplicar este método de restricción de dominio al campo de la agregación de lógicas.

\section{Agregación de lógicas}

En esta sección, investigaremos métodos para pasar de lógicas aceptadas individualmente a lógicas aceptadas colectivamente. Para eso, es conveniente pensar en escenarios donde distintos agentes aceptan ciertos principios o reglas lógicas. Si eso sucede, ¿qué mecanismos podemos adoptar para determinar la lógica adoptada por un grupo? Tal como sucede en la agregación de juicios, en la agregación de lógicas pueden estudiarse las propiedades de los distintos métodos de agregación.

Un problema metodológico natural de estos métodos es que la teoría de agregación que presentamos está basada en la lógica clásica. Entonces los lógicos no-clásicos deberán encarar la discusión metalógica de forma clásica. Esto es conceptualmente problemático, aunque ciertamente muy común. Los principales enfoques no-clásicos se construyen en metalenguajes clásicos. Tal es el caso de las lógicas multi-valuadas, y de las semánticas más usuales para las lógicas intuicionistas (semántica kripkeana con mundos posibles) y relevantes (semántica de Routley-Meyer con mundos posibles y accesibilidad ternaria). El desafío de elaborar meta-lógicas no-clásicas para las lógicas no-clásicas recientemente ha suscitado atención (véase Weber et al 2015), aunque es minoritario y bastante incipiente. El desarrollo de sistemas no-clásicos de agregación de juicios es un área prometedora y poco explorada. Sin embargo, para entender el fenómeno del desacuerdo lógico no sería muy útil: si los no-clásicos adoptaran una lógica no-clásica en el metalenguaje, la discusión (e incluso la comparación) entre sistemas clásicos y no-clásicos sería difícil, si no imposible.

El lenguaje $\mathcal{L}$ de nuestra teoría se basa en el lenguaje proposicional, pero incluye los símbolos primitivos $\ll \vDash »$ y $« \not \models »$, que representan la validez y la invalidez, y los símbolos $\ll\{\ldots\} »$, para expresar conjuntos. Las fórmulas de nuestro lenguaje pueden elaborarse del siguiente modo:

Regla de formación. Si $\phi_{1}, \ldots, \phi_{n}, \psi$ son fórmulas bien formadas de la lógica proposicional clásica, entonces $\ll\left\{\phi_{1}, \ldots, \phi_{n}\right\} \vDash \psi \gg$ y $\ll\left\{\phi_{1}, \ldots, \phi_{n}\right\} \not \models \psi »$ son fórmulas bien formadas de $\mathcal{L}$. Esto también abarca casos de inferencias sin premisas, como « $\psi \psi$. 7

7 Es posible extender la teoría a lenguajes de primer orden. Nos hemos restringido a los proposicionales por razones de simplicidad. Por otro lado, para simplificar a veces omitiré las llaves en las premisas. 
Por ejemplo, $\mathcal{L}$ incluye a los enunciados:

$$
\begin{gathered}
\{p, r \rightarrow s\} \not \models t \vee \neg \neg t \\
\{t \wedge s, \neg p, \neg \neg r\} \vDash t \wedge(q \rightarrow \neg \neg s) \\
\vDash p \vee(p \rightarrow q) \\
\text { etc. }^{8}
\end{gathered}
$$

Llamaremos enunciados lógicos a las fórmulas de $\mathcal{L}$. En este contexto, una agenda es un conjunto de enunciados lógicos que está cerrado bajo negación (es decir, se vota sobre $\Gamma \vDash \varphi$ sii se vota sobre $\Gamma \not \models \varphi)^{9}$.

Un conjunto de aceptación es un subconjunto de la agenda, que es Completo (incluye $\Gamma \vDash \varphi$ о $\Gamma \not \models \varphi$ para cada enunciado de la agenda) y Consistente (no puede incluir $\Gamma \vDash \varphi$ y $\Gamma \not \models \varphi)$. Además, los conjuntos de aceptación son estructurales, es decir que cumplen:

— Reflexividad: Si $\varphi \vDash \varphi$ está en la agenda, entonces se acepta $\varphi \vDash \varphi$.

- Monotonía: si se acepta $\Gamma \vDash \varphi$, y $\Gamma \cup \Delta \vDash \varphi$ está en la agenda, entonces se acepta $\Gamma \cup \Delta \vDash \varphi$.

— Transitividad: si se acepta que $\Gamma \vDash \varphi$ y que $\varphi \vDash \psi$, y $\Gamma \vDash \psi$ está en la agenda, entonces se acepta que $\Gamma \vDash \psi$.

Una cuarta característica que podría agregarse es la sustitutividad (usualmente llamada «estructuralidad», pero la llamamos distinto para evitar ambigüedades). Sea $s$ una función de sustitución de fórmulas de $L$ a fórmulas de $L$ tal que $s(p)$ es arbitrario para cualquier letra proposicional $p, s(\neg A)=\neg s(A)$, y $s\left(A^{*} B\right)=s(\mathrm{~A}) * s(B)$ para cualquier fórmula compuesta con conectivos binarios *. Entonces la propiedad de sustitutividad nos dice que, si se acepta que $\varphi_{1}, \ldots, \varphi_{n} \vDash \psi$, también se acepta que $s\left(\varphi_{1}\right), \ldots, s\left(\varphi_{n}\right) \vDash s(\psi)$. Por ejemplo, si se acepta que $p \vDash p \vee q$, también se acepta que $r \rightarrow r \vDash(r \rightarrow r) \vee \neg \neg t$. No trabajaremos específicamente con esta propiedad, aunque todos los resultados y ejemplos desarrollados a lo largo del artículo podrían obtenerse si asumimos que los individuos cumplen con ella.

Las definiciones de perfil y función de agregación son iguales a las de la sección anterior. Es decir, un perfil $P$ es un conjunto ordenado de conjuntos de aceptación $A_{i}$, que expresa la perspectiva lógica de un conjunto de individuos en determinado momento. Una función de agregación es una función que va de perfiles a subconjuntos de la agenda.

Siguiendo la estrategia de la sección anterior, por razones metodológicas (para que sea más fácil expresar los resultados formales a los que llegaremos), no suponemos nada más sobre el conjunto resultante de esta función (o «conjunto colectivo»). Es útil definir estas dos condiciones ${ }^{10}$ :

8 Es importante remarcar que la agenda incluye enunciados simples, donde el signo de implicación aparece una vez solamente, como $\ll \Gamma \vDash \phi » 0 \ll \Gamma \not \models \phi »$. No incluye enunciados más complejos, como $\ll \Gamma \vDash \phi \wedge \Gamma \not \models \psi \gg$.

9 En este contexto, asumimos que $\neg(\Gamma \vDash A)=(\Gamma \not \models A)$, mientras que $\neg(\Gamma \not \models A)=(\Gamma \vDash A)$.

10 Estas condiciones se definen de manera idéntica a las condiciones homónimas de la agregación de juicios. Sin embargo, conviene volver a formularlas, dado que el concepto de «conjunto de aceptación» es distinto (incluye la propiedad de estructuralidad, ausente en los conjuntos de aceptación de juicios). 
(Racionalidad Colectiva). Una función cumple racionalidad colectiva cuando $f\left(A_{1}, \ldots, A_{n}\right)$ es un posible conjunto de aceptación (i.e. consistente, completo y estructural).

(Dominio irrestricto). Una función cumple dominio irrestricto si su dominio incluye todos los perfiles (es decir, sólo suponemos que los agentes cumplen consistencia, completitud y estructuralidad).

Ahora, es interesante investigar qué métodos de agregación de lógicas se pueden utilizar, y cuáles son las propiedades de cada uno de estos métodos.

El método de unanimidad dice que el grupo acepta un enunciado lógico (de validez o invalidez) sii todos los individuos del grupo lo aceptan (la expresión formal de este método puede encontrarse en la sección anterior). Este método es conceptualmente sencillo, pero su aplicación es poco prometedora, porque hay un alto riesgo de que el grupo termine tomando muy pocas decisiones colectivas. Incluso en casos en donde el acuerdo sea muy general, supongamos con muchos agentes que acepten la lógica $S$, y un solo agente a que acepte una lógica distinta $S$, el grupo aceptará como válidas las inferencias válidas en $S$ y $S$, aceptará como inválidas las inferencias inválidas en $S$ y $S$, pero no se pronunciará sobre aquellas inferencias en donde $S$ y $S^{\prime}$ desacuerdan. Obviamente, la lógica colectiva no será completa relativamente a la agenda, dada la existencia de enunciados lógicos sobre los que el grupo no tiene un acuerdo unánime.

Uno podría cambiar la regla de unanimidad por la de unanimidad positiva. Según esta regla, el grupo acepta un enunciado lógico positivo (i.e. $\Gamma \vDash \varphi$ ) sii todo individuo lo hace, mientras que el grupo acepta un enunciado lógico negativo (i.e. $\Gamma \not \models \varphi$ ) sii algún individuo lo hace. Esta función cumplirá con racionalidad colectiva:

Teorema 7. Unanimidad positiva cumple con racionalidad colectiva.

Prueba. Sea $G$ el resultado de la función $f\left(A_{1}, \ldots, A_{n}\right)$, donde $f$ es la regla de Unanimidad positiva.

Consistencia y Completitud se siguen por construcción.

(Reflexividad). Todos los agentes aceptan $\phi \vDash \phi$, porque cumplen Reflexividad. Por ende, $G$ acepta $\phi \vDash \phi$.

(Monotonía). Supongamos que $G$ acepta $\Gamma \vDash \phi$. Entonces todos los agentes aceptan $\Gamma \vDash \phi$. Por ende, todos los agentes aceptan $\Gamma \cup \Delta \vDash \phi$, si $\Gamma \cup \Delta \vDash \phi$ está en la agenda (dado que cumplen Monotonía). Por lo tanto, $G$ acepta $\Gamma \cup \Delta \vDash \phi$.

(Transitividad). Supongamos que $G$ acepta $\Gamma \vDash \phi$ y $\phi \vDash \psi$. Entonces todos los agentes aceptan estos enunciados lógicos. En caso de que $\Gamma \vDash \psi$ esté en la agenda, todos los agentes lo aceptan, porque cumplen Transitividad. Por ende, $G$ acepta $\Gamma \vDash \psi$.

El problema del método de unanimidad positiva es que los defensores de lógicas más débiles tendrán el poder de debilitar la lógica aceptada colectivamente. Por ejemplo, si muchos agentes aceptan la lógica $S$, y un solo agente acepta una lógica más débil $S$, el grupo se inclinará hacia la lógica $S^{\prime}$.

Ahora podemos introducir la regla de la mayoría. Según la regla de la mayoría, un enunciado lógico (de validez o invalidez) se acepta colectivamente sii la mayoría de los individuos lo aceptan. Esta regla es mucho más prometedora que las anteriores, porque la existencia de individuos excepcionales no romperá con los consensos amplios no-unánimes. Además, se garantiza la Completitud de la lógica colectiva, cuando el grupo tiene un número impar de 
miembros: dada la completitud de los conjuntos de aceptación individuales, la mayoría necesariamente aceptará $\Gamma \vDash \phi$ o $\Gamma \not \models \phi$. También se garantiza la Consistencia, pues no puede suceder que la mayoría acepte $\Gamma \vDash \phi$ y también $\Gamma \not \models \phi$.

Queda por establecer un asunto más importante: si la regla de mayoría cumple con Racionalidad Colectiva. Es decir, si esta regla garantiza la estructuralidad de la lógica grupal. En verdad, conviene analizar caso por caso, porque no sucede lo mismo con las distintas propiedades estructurales. Investigaremos si, al utilizar la regla de la mayoría, las propiedades estructurales de los conjuntos de aceptación individuales se preservan al conjunto colectivo.

Naturalmente, la reflexividad va a preservarse:

Teorema 8. La regla de la mayoría preserva Reflexividad.

Prueba. Todos los agentes aceptan $\varphi \vDash \varphi$ (porque cumplen Reflexividad), entonces esto también sucede para la mayoría de los agentes. Por lo tanto, el criterio de mayoría resultará en una lógica donde $\varphi \vDash \varphi$ para toda oración $\varphi$.

Una prueba también sencilla se puede dar para Monotonía:

Teorema 9. La regla de la mayoría preserva monotonía.

Prueba. Supongamos que el grupo acepta $\Gamma \vDash \varphi$. Por lo tanto, para la mayoría de los agentes, $\Gamma \vDash \varphi$. Entonces, como cada agente cumple monotonía, para la mayoría de los agentes también $\Gamma \cup \Delta \vDash \varphi$, en caso de que $\Gamma \cup \Delta \vDash \varphi$ esté en agenda. Por ende, el grupo acepta $\Gamma \cup \Delta \vDash \varphi$.

Ahora bien, es interesante observar que la propiedad de transitividad no se preserva bajo el voto mayoritario.

Teorema 10. Si la agenda involucra tres enunciados lógicos del tipo $\varphi \vDash \psi, \psi \vDash \chi, \varphi \vDash \chi$, siendo $\varphi, \psi$ y $\chi$ oraciones distintas de la lógica proposicional, y la cantidad de votantes es impar y mayor o igual a 3, el voto por mayoría en un dominio irrestricto no preserva transitividad.

Prueba. Como el dominio es irrestricto, puede darse el siguiente perfil respecto a estos tres enunciados lógicos:

$\begin{array}{llll}\text { Lógica 1: } & \phi \vDash \psi & \psi \vDash \chi & \phi \vDash \chi \\ \text { Lógica 2: } & \phi \vDash \psi & \psi \neq \chi & \phi \not \models \chi \\ \text { Lógica 3: } & \phi \not \models \psi & \psi \vDash \chi & \phi \not \models \chi \\ \text {.. } & & & \\ \text { Lógica par: } & \phi \vDash \psi & \psi \not \models \chi & \phi \not \models \chi \\ \text { Lógica impar: } & \phi \not \models \psi & \psi \vDash \chi & \phi \not \models \chi \\ \text {... } & & & \\ \text { Mayoría: } & \phi \vDash \psi & \psi \vDash \chi & \phi \not \models \chi\end{array}$

En este escenario, el conjunto de inferencias aceptado por cada agente es transitivo, pero el voto mayoritario arroja un conjunto no-transitivo. El caso más sencillo es cuando hay 3 agentes; agregar agentes de a dos (uno par y otro impar) de la manera especificada mantiene las mayorías anteriores.

También para este caso, podemos adaptar el resultado de Dietrich y List (2007), cambiando un poco la terminología. En este caso, la propiedad deseable en conjunto resultante no es sólo la consistencia y la completitud, sino también la estructuralidad (en particular, la 
transitividad). Es fácil ver que si el dominio es irrectricto, una agenda con tres oraciones de la lógica proposicional distintas y sus respectivas relaciones de validez e invalidez (es decir, asumiendo la «completitud» de la agenda) tiene una propiedad análoga a estar mínimamente conectada (diremos que está «lógicamente conectada»). Para definir la conexión lógica, hace falta definir qué es un conjunto mínimamente ilógico (el análogo a un conjunto mínimamente inconsistente).

Definición 11. Un conjunto $X$ de enunciados lógicos es ilógico sii tiene alguna de las siguientes fallas lógicas:

i. $X$ incluye $\Gamma \vDash \phi$ у $\Gamma \not \models \phi$ (falla de Consistencia)

ii. $X$ incluye $\phi \not \models \phi$ (falla de Reflexividad)

iii. $X$ incluye $\Gamma \vDash \phi$ у $\Gamma \cup \Delta \not \models \phi$ (falla de Monotonía)

iv. $X$ incluye $\Gamma \vDash \phi, \phi \vDash \psi$ y $\Gamma \not \models \psi($ falla de Transitividad)

(a) Un conjunto $X$ de enunciados lógicos es mínimamente ilógico sii es ilógico, pero para cualquier $Y \subset X, Y$ no es ilógico.

Definición 12. Un conjunto $X$ de enunciados lógicos está lógicamente conectado sii (a) contiene un conjunto $Z$ mínimamente ilógico tal que $|Z| \geq 3$, y (b) contiene un conjunto $Z^{*}$ mínimamente ilógico y existe un conjunto $Y \subseteq Z^{*}$ de cardinalidad par, tal que $\left(Z^{*}-Y\right) \cup\{\neg z \mid z \in Y\}$ no es ilógico.

Lema 13. Sea $X$ una agenda que incluye el conjunto $\pm\{\phi \vDash \psi, \psi \vDash \chi, \phi \vDash \chi\}$, siendo $\phi, \psi$ y $\chi$ tres oraciones proposicionales distintas. La agenda $X$ está lógicamente conectada, por el subconjunto $Z=\{\phi \vDash \psi, \psi \vDash \chi, \phi \not \models \chi$.

Prueba. (a) Puede observarse que $Z=\{\varphi \vDash \psi, \psi \vDash \chi, \varphi \not \models \chi\}$ tiene la falla de Transitividad, por lo cual es ilógico. Sin embargo, ninguno de sus subconjuntos propios tiene esa falla (para eso necesitaría tener tres enunciados). Por otro lado, $Z$ no tiene la falla de Reflexividad, porque no contiene enunciados del tipo $\varphi \not \models \varphi$. Es fácil ver que $Z$ tampoco tiene una falla de Consistencia, porque toma sólo un elemento de cada par de validez/invalidez de la agenda. Trivialmente $Z$ tampoco tiene la falla de Monotonía: los tres enunciados tienen conclusiones distintas. Naturalmente, si no hay falla de Monotonía, Consistencia o Reflexividad en $Z$, tampoco la hay en sus subconjuntos propios. Por ende, los subconjuntos propios de $Z$ no son ilógicos. Esto implica que $Z$ es mínimamente ilógico.

(b) El subconjunto $\{\varphi \vDash \psi, \psi \vDash \chi\}$ puede sustraerse de $Z$, y cambiarse por $\{\varphi \not \models \psi, \psi \not \models \chi\}$, resultando en el conjunto transitivo $Z^{\prime}=\{\varphi \not \models \psi, \psi \not \models \chi, \varphi \not \models \chi\}$. Por las mismas razones que $Z, Z$ no tiene fallas de Consistencia, Monotonía o Reflexividad. Por ende, $Z$ no es ilógico.

Por (a) y (b), $X$ es una agenda lógicamente conectada.

Con este resultado, podemos adaptar el teorema 5 de la sección anterior para afirmar que si el dominio es irrestricto y la agenda contiene $\pm\{\phi \vDash \psi, \psi \vDash \chi, \phi \vDash \chi\}$, entonces la regla de mayoría (o cualquier otra regla no-dictatorial que cumpla unanimidad y sistematicidad) no puede cumplir con Racionalidad Colectiva ${ }^{11}$.

Es importante observar que la paradoja discursiva para la validez no es un resultado totalmente abstracto. El perfil que genera la paradoja no es particularmente extraño ni arbi-

11 Omito la prueba aquí, pero es estructuralmente idéntica a la prueba de List (2012). Un ejercicio similar es el de Dietrich (2007), que ha extendido el resultado de imposibilidad a otras lógicas no-clásicas, usando siempre el concepto de «conjunto mínimamente conectado». 
trario. De hecho, si tomamos tres lógicas bastante utilizadas, como $L P, K 3$ y la lógica clásica, podemos instanciar la paradoja.

Ejemplo 14. Supongamos que la agenda incluye el siguiente conjunto de enunciados lógicos:

$$
\pm\{p, p \rightarrow q \vDash q ; \quad q \vDash q \wedge(r \vee \neg r) ; \quad p, p \rightarrow q \vDash q \wedge(r \vee \neg r)\}
$$

Ahora pensemos en el siguiente perfil:

Grupo: $\{1,2,3\}$

Perfil: <Lógica clásica, $K 3, L P>$

\begin{tabular}{|c|c|c|c|}
\hline 1-Clásica: & $p, p \rightarrow q \vDash q$ & $q \vDash q \wedge(r \vee \neg r)$ & $p, p \rightarrow q \vDash q \wedge(r \vee \neg r)$ \\
\hline$-K 3:$ & $p, p \rightarrow q \vDash q$ & $q \not \models q \wedge(r \vee \neg r)$ & $p, p \rightarrow q \not \models q \wedge(r \vee \neg$ \\
\hline$-L P:$ & $p, p \rightarrow q \not \neq q$ & $q \vDash q \wedge(r \vee \neg r)$ & $p, p \rightarrow q \not \vDash q \wedge(r \vee$ \\
\hline Mayoria: & $p, p \rightarrow q \vDash q$ & $q \vDash q \wedge(r \vee \neg r)$ & $p, p \rightarrow q \not \models c$ \\
\hline
\end{tabular}

Es fácil ver que estas tres lógicas constituyen conjuntos individuales de aceptación, pero la regla de la mayoría no satisface Racionalidad Colectiva (porque no preserva la transitividad).

Así como sucedía con la agregación de juicios, este nuevo «dilema discursivo» tiene distintas respuestas. Una manera un tanto extrema de responder a este dilema es usar el resultado a favor de una lógica no-transitiva, como la desarrollada recientemente por Cobreros et al. (2013). Más adelante, evaluaremos esta clase de propuestas, en particular para la discusión sobre paradojas semánticas.

Otra manera de responder al dilema es adoptar la regla de premisas. Esto no parece ser más que un recurso técnico ad hoc, puesto que el caso en cuestión no tiene la estructura premisas-conclusión. En lógica, el hecho de que $\varphi \vDash \psi \mathrm{y} \psi \vDash \chi$ no siempre es la razón para aceptar que $\varphi \vDash \chi$. Pensemos en el caso de la lógica clásica, donde se dan los siguientes tres casos de validez:

1. $p \wedge \neg p \vDash q \wedge s$

2. $q \wedge s \vDash r \vee \neg r$

3. $p \wedge \neg p \vDash r \vee \neg r$.

No diríamos en ese caso que las primeras dos afirmaciones funcionan como «premisas» para la última afirmación. Ciertamente, en un planteo puramente deductivo, la regla de Corte nos permitiría inferir (3) a partir de (1) y (2). Pero en las lógicas donde vale (3), podemos obtener (3) sin necesidad de obtener (1) o (2). En ese sentido, hay una clara diferencia entre este caso y el caso de $\{p, q,(p \wedge q)\}$.

\section{Restricciones de dominio}

Uno podría pensar que si rechazamos Dominio Irrestricto y ponemos ciertas restricciones a los perfiles aceptados, podemos evitar el resultado de imposibilidad de la sección anterior. Una estrategia prometedora es utilizar la noción de alineación uniforme de List y Pettit (2002). De hecho, podemos probar un resultado análogo al Teorema 6 para la agregación de enunciados lógicos. Para eso es necesario definir un concepto: 
(Inclusión). Decimos que $L_{1} \subseteq L_{2}$ cuando, siempre que $\Gamma \vDash \mathrm{A}$ sea aceptado por $L_{1}$, también es aceptado por $L_{2}{ }^{12}$

Ahora podemos pasar al resultado:

Teorema 15. Si el grupo $G$ tiene un número impar $n$ de miembros, y todo perfil puede ponerse en cadena de modo que $L_{1} \subseteq L_{2} \subseteq \ldots \subseteq L_{n}$, entonces la regla de mayoría preserva transitividad (y por ende, cumple Racionalidad Colectiva).

Prueba. Si suponemos que, en todo perfil, una lógica está incluida en la otra, entonces necesariamente las lógicas se pueden alinear uniformemente en cualquier perfil como en el siguiente cuadro (ejemplifico con 3 lógicas, pero puede extenderse naturalmente a cualquier número impar):

\begin{tabular}{lcccc}
\hline & Lógica 1 & Lógica 2 & Lógica 3 & Mayoría \\
\hline Inferencia 1 & No & Sí & Sí & Sí \\
Inferencia 2 & Sí & Sí & Sí & Sí \\
Inferencia 3 & No & No & No & NO \\
\hline
\end{tabular}

Esto garantiza la transitividad de la lógica colectiva (y por ende, su estructuralidad). De hecho, la mayoría va a votar aquello que vota la lógica que queda en el medio de la tabla (en este caso la lógica 2): si el votante medio acepta la inferencia $I$, una mayoría (conformada por el votante medio y los votantes a su derecha) acepta $I$; en cambio, si el votante medio rechaza $I$, una mayoría rechaza $I$ (conformada por el votante medio y los votantes a su izquierda). Esto no constituye una dictadura, porque distintas personas pueden ser el votante medio en distintos perfiles.

Ejemplo 16. Un ejemplo de lo anterior es el perfil $\angle F D E, L P$, Lógica clásica $>$, dado que $F D E \subseteq L P \subseteq$ Lógica clásica. El voto por mayoría, siguiendo los resultados anteriores, arrojará necesariamente $L P$, es decir, el votante medio.

El alcance de esta restricción puede parecer demasiado acotado. Pero el resultado anterior se puede extender. La prueba no necesita que las lógicas estén incluidas en cadena, sino algo un poco más débil:

Teorema 17. Sea $G$ un grupo con un número impar $n$ de miembros. Si para todo perfil, la clase de inferencias puede partirse en dos, y una clase es tal que $L_{1} \subseteq L_{2} \subseteq \ldots \subseteq L_{n}$, mientras que la otra clase es tal que $L_{n} \subseteq \ldots L_{2} \subseteq L_{1}$, entonces la regla de la mayoría preserva transitividad (y por ende, satisface Racionalidad Colectiva).

Prueba Si esto sucede, en cualquier perfil tenemos una alineación uniforme del tipo que describen List y Pettit (2002), donde los que aceptan una inferencia están en bloque, todos a la derecha o a la izquierda de los que la rechazan. Imaginemos el siguiente perfil, donde las inferencias se separan en dos clases disyuntas. La clase $A$ es tal que $L_{1} \subseteq L_{2} \subseteq L_{3}$, y la clase $B$ es tal que $L_{3} \subseteq L_{2} \subseteq L_{1}$ (ejemplifico con 3 lógicas, pero el argumento puede extenderse fácilmente a cualquier número impar). Supongamos que las inferencias impares son de la clase $A$, y las pares son de la clase $B$. El diagrama queda como sigue:

12 Nótese que la inclusión no se pronuncia sobre los enunciados de invalidez. De cualquier manera, dado que los agentes tienen conjuntos completos de aceptación, lo que no consideran válido lo considerarán inválido. Por ende, si $L_{1} \subseteq L_{2}$, entonces lo que es inválido para $L_{2}$ también es inválido para $L_{1}$. 


\begin{tabular}{lcccc}
\hline & Lógica 1 & Lógica 2 & Lógica 3 & Mayoría \\
\hline Inferencia 1 (A) & No & Sí & Sí & SÍ \\
Inferencia 2 (B) & Sí & Sí & No & SÍ \\
Inferencia 3 (A) & No & No & Sí & NO \\
Inferencia 4 (B) & Sí & No & No & NO \\
\hline
\end{tabular}

Es fácil notar que la mayoría se inclina por lo que vota la lógica del medio (en este caso, la lógica 2), por razones similares a las del teorema 15. Esto garantiza la transitividad (y también la estructuralidad) del voto mayoritario. Y no constituye una dictadura, porque distintas personas pueden ser el votante medio en distintos perfiles.

A continuación, exploraremos perfiles un poco más complejos. Usaremos el concepto de unión e intersección de lógicas. La unión entre dos lógicas $L$ y $L^{\prime}, L \cup L^{\prime}$, es la lógica $L^{*}$ que valida solamente las inferencias validadas por $L$ o por $L^{\prime}$ (e invalida el resto $)^{13}$. En cambio, la intersección entre dos lógicas $L$ y $L^{\prime}, L \cap L^{\prime}$, es la lógica $L^{*}$ que valida solamente las inferencias validadas tanto por $L$ como por $L^{\prime}$ (e invalida al resto).

A partir de estos dos conceptos, podemos ejemplificar el teorema 17 con el siguiente conjunto de lógicas.

Ejemplo 18. La lógica deMorgan débil, llamada $S 3$, es la intersección entre $K 3$ y $L P$. El perfil $\langle S 3, K 3, L P\rangle$ necesariamente preserva transitividad cuando aplicamos el criterio de mayoría. De hecho, podemos diferenciar dos clases: las inferencias válidas en $K 3$ (como el Modus Ponens), y las que no son válidas en $K 3$ (como el Tercero Excluido). Respecto a la primera clase, sucede que $L P=S 3 \subseteq K 3$. Respecto a la segunda clase, sucede que $K 3=S 3 \subseteq L P$. Naturalmente, el voto por mayoría arrojará los mismos resultados que la lógica $S 3$, el «votante medio».

Las intersecciones entre lógicas tienen cierta importancia en este contexto. En primer lugar, porque el ejemplo anterior puede repetirse para cualquier conjunto de tres lógicas, donde una es intersección de las otras dos. En segundo lugar, porque incluso cuando esto no sucede, y asumimos simplemente que $L_{1} \subseteq L_{2}$ y $L_{1} \subseteq L_{3}$, la votación mayoritaria arroja la intersección entre dos de esas lógicas, $L_{2}$ y $L_{3}$, sin que necesariamente esa intersección sea idéntica a $L_{1}$. La votación mayoritaria arrojaría en ese caso una lógica estructural nueva (no sostenida por ninguno de los agentes). Este resultado puede ser probado:

Teorema 19. Sea $<L_{1}, L_{2}, L_{3}>$ un perfil donde $L_{1} \subseteq L_{2}$ y $L_{1} \subseteq L_{3}$. El voto mayoritario arrojará la intersección $S$ entre $L_{2}$ y $L_{3}$.

Prueba. Supongamos que la inferencia $I$ es válida en $S$. Entonces, es válida en $L_{2}$ y $L_{3}$. Por ende, es válida en el voto de mayoría. Por otro lado, supongamos que la inferencia $I$ no es válida en $S$. Entonces, no es válida en $L_{2}$ (o en $L_{3}$, por simetría). Por ende, no es tampoco válida en $L_{1}$. Entonces, no es válida en el voto por mayoría.

13 El concepto de unión guarda similitud con la noción de splicing o combinación desarrollada en Carnielli et al. (2008). Sin embargo, la unión y la combinación son distintas (podría decirse que la combinación es matemáticamente más elegante). En particular, la combinación o splicing toma la unión de los axiomas y acepta las consecuencias de esa unión de axiomas: en ese sentido, la combinación de dos lógicas puede validar inferencias que ninguna de las dos lógicas validaba por separado. 
Este teorema tiene una consecuencia bastante positiva, que es otro resultado de posibilidad. El resultado se basa en el siguiente lema:

Lema 20. Si $L_{1}$ y $L_{2}$ son lógicas estructurales, y $S$ es su intersección, entonces $S$ es estructural.

Prueba. Se deriva del Teorema 7, dado que la intersección entre lógicas tiene el mismo resultado que el voto por Unanimidad positiva.

Entonces podemos establecer lo siguiente:

Teorema 21. Sea $\mathrm{G}$ un grupo de tres agentes. Si todo perfil puede ordenarse de modo tal que $L_{1} \subseteq L_{2}$ y $L_{1} \subseteq L_{3}$, entonces la regla de la mayoría preserva transitividad (y por ende, satisface Racionalidad Colectiva).

Prueba. Por el Teorema 19 y el Lema 20.

Podemos decir esto de otro modo. En determinados escenarios las lógicas no están «encadenadas», pero aún así podemos preservar la estructuralidad por medio de un voto mayoritario. Estos casos no son extraños: muchas discusiones filosóficas tienen esta estructura, con dos agentes (o conjuntos de agentes) que aceptan posiciones distintas entre sí, y otro agente (o conjunto de agentes) que acepta una posición más débil que esas dos. Si este desacuerdo fuera lógico, sabemos que el voto mayoritario no necesariamente corresponderá con ninguna de las lógicas de los agentes, pero será un sistema estructural.

Vale remarcar que el teorema 21 no se deriva del teorema sobre alineación uniforme. Porque hay perfiles en que $L_{1} \subseteq L_{2}$ y $L_{1} \subseteq L_{3}$, pero no podemos lograr una alineación uniforme. Un ejemplo es tomar $F D E, K 3$ y $L P$, donde sabemos que $F D E \subseteq K 3$ y $F D E \subseteq L P$, y las siguientes inferencias:

\begin{tabular}{lccc}
\hline & $F D E$ & $K 3$ & $L P$ \\
\hline$p, p \rightarrow q \vDash q$ & No & Sí & No \\
$\vDash p \vee \neg p$ & No & No & Sí \\
$p \wedge \neg p \vDash q \vee \neg q$ & No & Sí & Sí \\
\hline
\end{tabular}

El lector puede comprobar que no hay manera de alinear uniformemente a estos agentes. Sin embargo, el voto por mayoría arrojará lo mismo que $S 3$. Es decir, de las inferencias en cuestión se aceptará solamente $p \wedge \neg p \vDash q \vee \neg q$.

Un resultado similar al teorema anterior (aunque con distintas consecuencias) se puede probar para el caso de dos lógicas y su unión:

Teorema 22. Sea $<L_{1}, L_{2}, L_{3}>$ un perfil donde $L_{1} \subseteq L_{3}$ y $L_{2} \subseteq L_{3}$. El resultado del voto mayoritario es la unión entre $L_{1}$ y $L_{2}$.

Prueba. Similar a la prueba del teorema 19.

Sin embargo, la unión de dos lógicas no preserva transitividad. El ejemplo 14 antes mencionado puede servir de ilustración: 


$$
\begin{array}{llll}
\text { K3: } & p, p \rightarrow q \vDash q & q \not \vDash q \wedge(r \vee \neg r) & p, p \rightarrow q \not \models q \wedge(r \vee \neg r) \\
\text { LP: } & p, p \rightarrow q \not \vDash q & q \vDash q \wedge(r \vee \neg r) & p, p \rightarrow q \not \vDash q \wedge(r \vee \neg r) \\
\text { K3 } \cup L P: & p, p \rightarrow q \vDash q & q \vDash q \wedge(r \vee \neg r) & p, p \rightarrow q \not \vDash q \wedge(r \vee \neg r)
\end{array}
$$

En este caso, es claro que dos lógicas son transitivas, pero su unión no lo es. Vale aclarar que, de todos modos, los resultados de imposibilidad no siempre se generan con uniones. Puede observarse el siguiente ejemplo:

Ejemplo 23

\begin{tabular}{llllll}
\hline Lógica 1: & $\phi \vDash \psi$ & $\psi \vDash \chi$ & $\phi \vDash \chi$ & $\mathrm{A} \vDash \mathrm{B}$ & $\mathrm{C} \not \models \mathrm{D}$ \\
Lógica 2: & $\phi \vDash \psi$ & $\psi \neq \chi$ & $\phi \not \models \chi$ & $\mathrm{A} \vDash \mathrm{B}$ & $\mathrm{C} \not \models \mathrm{D}$ \\
Lógica 3: & $\phi \not \models \psi$ & $\psi \vDash \chi$ & $\phi \not \models \chi$ & $\mathrm{A} \not \models \mathrm{B}$ & $\mathrm{C} \vDash \mathrm{D}$ \\
Mayoria: & $\phi \vDash \psi$ & $\psi \vDash \chi$ & $\phi \not \models \chi$ & $\mathrm{A} \vDash \mathrm{B}$ & $\mathrm{C} \not \models \mathrm{D}$ \\
\hline
\end{tabular}

Aquí, el voto por mayoría arroja un conjunto no-estructural. Sin embargo, este conjunto colectivo no puede formarse con la unión entre dos de las lógicas del grupo.

Antes de pasar al próximo apartado, recapitulemos los resultados obtenidos hasta aquí. Por un lado, pudimos probar resultados de imposibilidad para la agregación de lógicas; en particular, la no-preservación de la Transitividad mediante la regla de la mayoría. Este resultado emula a la paradoja discursiva, que mostraba que la consistencia no se preservaba mediante la regla de la mayoría, y de hecho en ambos casos las agendas tienen características similares (la agenda está mínimamente conectada en el caso original, y «lógicamente» conectada en el caso de la agregación de lógicas). Por otro lado, pudimos adaptar algunos resultados de posibilidad, en particular aquellos relacionados con la noción de Alineación Uniforme de List y Pettit (2002). Mencionamos tres escenarios en particular que nos garantizan el éxito de la regla de la mayoría: en primer lugar, los perfiles en que las lógicas están encadenadas, y una está incluida en la otra (como en el Teorema 15); en segundo lugar, los perfiles en que las inferencias pueden partirse en dos conjuntos, respecto a las cuales hay dos cadenas inversas (como en el Teorema 17); en tercer lugar, los perfiles en que las lógicas tienen una estructura triangular «en $\mathrm{V} »$, donde dos lógicas distintas incluyen una tercera lógica (como en el Teorema 21).

\section{Algunas aplicaciones de la agregación lógica}

Como mencioné en la introducción, uno de los casos donde el desacuerdo lógico parece irresoluble es el de las paradojas semánticas, donde existen muchas posiciones en conflicto y no parece haber elementos que nos permitan zanjar la discusión. En esta sección aplicaremos algunos de los conceptos desarrollados hasta aquí al campo de las paradojas semánticas.

Supongamos que, por diagonalización, existe una oración $\lambda$ que tiene necesariamente el mismo valor de verdad que $\neg T(\langle\lambda\rangle)$. También vale Intersustitutividad, por lo cual $A \mathrm{y}$ $T(<A>)$ son intersustituibles en cualquier oración, en un contexto no-opaco. El argumento de la paradoja del Mentiroso nos muestra que, bajo esos supuestos, obtenemos un sistema trivial. La paradoja puede derivarse así: 
1. $\mid \lambda$

2. $\mid T(<\lambda>) \quad$ Intersustitutividad, 1

3. $\mid \neg T(<\lambda>)$ Diagonalización, 1

4. $\mid \perp \quad 2,3$

5. $\neg \lambda \quad$ Introducción de la negación, 1-4

6. $\neg \mathrm{T}(<\lambda>)$ Intersustitutividad, 5

7. $\lambda$ Diagonalización, 6

8. $\perp \quad 5,7$

9. B Explosión, 8

Los pasos subrayados expresan inferencias que son muchas veces rechazadas. La introducción de la negación es rechazada por los que sostienen lógicas paracompletas (donde ni $A$ ni $\neg A$ podrían ser verdaderas), como Kripke (1975). Mientras que Explosión es rechazada por los que sostienen lógicas paraconsistentes, como Priest (2006). Las posiciones clásicas o tarskianas rechazan, por su parte, Intersustitutividad.

Para aplicar la agregación a estos debates debemos realizar algunas modificaciones al esquema anterior. Primero, en la agregación de teorías de la verdad ya no usamos sistemas «puros» como $K 3$ o LP, sino sistemas aumentados con un mecanismo de auto-referencia, un predicado de verdad y reglas para ese predicado (como Intersustitutividad). Llamamos a estos sistemas $\mathrm{K}^{+}, \mathrm{LP}^{+}$, etc.; y también diremos que son «lógicas» a pesar de que en sentido estricto son teorías de la verdad (es decir, lógicas suplementadas con principios para la verdad). En segundo lugar, para poder obtener resultados interesantes, nos enfocamos en casos donde hay una igualdad de condiciones entre distintas propuestas; en esos casos, asumimos que no importa cuántos agentes aceptan cada lógica, siguiendo las ideas de Kelly (2010) sobre el desacuerdo entre pares. Entonces, hacemos de cuenta que cada lógica es un agente, y que hay tantos agentes como lógicas aceptables en el debate (más adelante, en las respuestas a objeciones, también veremos qué pasa si prescindimos de este supuesto). Si complementamos esto con el punto anterior, diremos que cada teoría de la verdad será un agente determinado.

Ahora veamos algunos resultados. Podríamos trazar el siguiente perfil de las posiciones más comunes en el debate, donde suponemos que «Tarskiano» es la teoría clásica $T B$ (donde no se admite la intersustitutividad), «Paracompleto» es $K 3^{+}$y «Paraconsistente» es $L P^{+}$:

\begin{tabular}{lcccc}
\hline & $\begin{array}{c}\text { Tarskiano } \\
(T B)\end{array}$ & $\begin{array}{c}\text { Paracompleto } \\
\left(K 3^{+}\right)\end{array}$ & $\begin{array}{c}\text { Paraconsistente } \\
\left(\mathbf{L P}^{+}\right)\end{array}$ & Mayoría \\
\hline Intersustitutividad & No & Sí & Sí & Sí \\
Explosión & Sí & Sí & No & Sí \\
Intro $\neg$ & Sí & No & Sí & Sí \\
\hline
\end{tabular}

En este caso, el voto mayoritario arroja un conjunto de principios que, a primera vista, no parecen conjuntamente posibles. Sin embargo, ahí es cuando aparecen nuestros resultados. Ciertamente, una manera de dar lugar al voto mayoritario es abandonar la transitividad. 
Recientemente, diversos autores han propuesto abandonar la transitividad para resolver las paradojas semánticas (Cobreros et al. 2013) ${ }^{14}$. Aquí podemos ver una posible motivación: esta es una manera de dar lugar a consensos mayoritarios en el debate sobre la paradoja del Mentiroso. De cualquier manera, la plausibilidad de este planteo depende de la relativa paridad entre (por ejemplo) las propuestas paracompletas, paraconsistentes y clásicas.

Sin embargo, hay otros escenarios, menos problemáticos pero igual de interesantes. Supongamos que la lógica clásica queda excluida por alguna razón (por ejemplo, porque Intersustitutividad se considera una propiedad esencial para el concepto de Verdad). El perfil podría incluir, no a un lógico clásico, sino a un lógico cuatrivalente (aquí asumimos que es $F D E)$. Entonces el escenario sería el siguiente:

\begin{tabular}{lcccc}
\hline & $\begin{array}{c}\text { Cuatrivalente } \\
\left(F D E^{+}\right)\end{array}$ & $\begin{array}{c}\text { Paracompleto } \\
\left(K 3^{+}\right)\end{array}$ & $\begin{array}{c}\text { Paraconsistente } \\
\left(\mathbf{L P}^{+}\right)\end{array}$ & Mayoría \\
\hline Intersustitutividad & Sí & Sí & Sí & Sí \\
Explosión & No & Sí & No & NO \\
Intro $\neg$ & No & No & Sí & NO \\
\hline
\end{tabular}

Es fácil establecer que las lógicas en cuestión tienen las siguientes relaciones de inclusión: $F D E^{+} \subseteq L P^{+}$y $F D E^{+} \subseteq K 3^{+}$. Entonces, en este caso el voto por mayoría arrojaría la intersección entre $L P^{+}$y $\mathrm{K3}^{+}$, que es $S 3^{+}$. $S 3^{+}$es muy similar, aunque no idéntica, a $F D E^{+}$: la inferencia $p \wedge \neg p \vDash q \vee \neg q$ es válida en $S 3^{+}$pero inválida en $F D E^{+}$. Como establecimos antes, la intersección entre $L P^{+}$y $K 3^{+}$, que son dos lógicas estructurales, es necesariamente una lógica estructural.

En este caso, son muy pocos los lógicos que han defendido $S 3^{+}$, y en general lo han hecho por razones técnicas (véase Horsten y Halbach 2006). Estas consideraciones a partir de la agregación de lógicas podrían servir como razón para adoptar esta lógica, generada del consenso entre diversas posiciones razonables.

Como indicó un réferi anónimo, la aplicación de estos resultados para la solución de paradojas semánticas es mucho más compleja de lo que presenté hasta ahora. Aquí me limité a considerar algunas propuestas multi-valuadas, pero hay decenas de posibilidades exploradas en la literatura sobre la paradoja del mentiroso, no sólo propuestas no-clásicas (como las lógicas del sinsentido, las revisionistas, las lógicas suplementadas con condicionales de Field, etc.), sino también propuestas clásicas que abandonan el principio de Intersubstitutividad (como casi todas las desarrolladas por Halbach (2011), entre ellas TPA, TPA|, PUTB, etc.). Para el debate sobre agregación de lógicas, naturalmente las propuestas que apelan a lógicas no-clásicas serán más interesantes; pero es cierto que una vez que los lenguajes son aumentados con aparatos aritméticos para lidiar con la auto-referencia, los principios sobre la verdad cobran igual importancia.

14 Vale aclarar que la lógica propuesta por estos autores, $S T^{+}$, no es la unión entre $K 3^{+}$y $L P^{+}$. Sin embargo, la unión entre $K^{+}$y $L P^{+}$está incluida en $S T^{+}$. En mi conocimiento, el único autor que ha propuesto algo similar a $K 3 \cup L P$, en otro contexto, fue Cook (2005:221). 
De todas formas, el propósito de esta sección no es explorar las numerosas variantes de agregación para resolver la paradoja del mentiroso, sino mostrar que el mecanismo de agregación puede ser útil, al menos en ciertos casos, para visualizar o justificar salidas al problema. Decidí presentar algunos casos interesantes donde la agregación hiciera una diferencia visible. Es posible realizar un análisis mucho más exhaustivo de las distintas teorías de la verdad y sus posibilidades de agregación, pero una investigación de ese tipo está por fuera del alcance de este artículo.

\section{Algunas objeciones al planteo}

\section{¿MISMO CRITERIO PARA TODOS LOS ENUNCIADOS LÓGICOS?}

Como un réferi anónimo indica, los mecanismos de agregación sobre agendas que describo en el artículo utilizan siempre la misma regla para todos los enunciados. Es decir, que si utilizo la regla de la mayoría para decidir sobre $\Gamma \vDash A$, debo utilizar el mismo criterio para decidir sobre $\Delta \vDash \mathrm{B}$.

Ciertamente, esa característica no aparece en todo mecanismo de agregación. Los mecanismos de agregación más sencillos aplican la misma «delimitación» para cada proposición, pero esto no sucede con las reglas llamadas quota rules. En estos mecanismos, cada proposición $p$ corresponde a un número $r_{\mathrm{p}}$, que llamamos «límite», entre 0 y 1 (usualmente $\left.0.5<r_{p} \leq 1\right)$. El grupo aceptará la proposición $p$ siempre y cuando el cociente de individuos que aceptan $p$ sobre individuos del grupo es mayor o igual al límite $r_{p}$.

Ciertamente, estas ideas parecen conceptualmente adecuadas para la agregación de lógicas: algunos principios podrían ser más estables que otros, de modo que es imposible abandonarlos a menos que exista unanimidad o algo cercano a eso. El problema es que la lógica resultante no es técnicamente muy promisoria: será reflexiva, pero podría no ser completa, contractiva (si el límite para $\Gamma, \mathrm{A} \vDash \mathrm{B}$ es mayor que el límite para $\Gamma, \mathrm{A}, \mathrm{A} \vDash \mathrm{B}$ ), monotónica (si el límite para $\Gamma \cup \Delta \vDash A$ es mayor que el límite para $\Gamma \vDash A$ ) ni transitiva. De todas formas, estos problemas podrían evitarse si establecemos restricciones artificiales de antemano sobre los límites (por ejemplo, que el límite para $\Gamma, A \vDash B$ no pueda ser mayor al límite para $\Gamma, \mathrm{A}, \mathrm{A} \vDash \mathrm{B})$.

\section{El DESACUERDo LÓGICO, ¿DESCRIPTIVO O EVALUATIVO?}

En la mayoría de los ejemplos de la literatura sobre agregación de juicios, el método se utiliza para desacuerdos de valor, donde las opiniones de los individuos tienen cierto peso. Es razonable que los españoles (por ejemplo) decidan cuál debe ser su salario mínimo; si todos los ciudadanos creen algo distinto a lo que sucede en el país, hay algo en el mecanismo democrático que está fallando. Pero el desacuerdo lógico tiene una naturaleza distinta: parece perfectamente posible que todo el mundo crea que A implica B, pero que eso sea falso. Las opiniones de los agentes no parecen especialmente relevantes para determinar si determinada inferencia es correcta o no.

Según la visión que adopto en este artículo, las discusiones más recientes sobre lógica tienden a probar que el desacuerdo lógico es más evaluativo que descriptivo, como sugiere Field (1998). No hay un hecho que pueda inclinar la balanza definitivamente entre $L P$ y $K 3$, por ejemplo. Todas esas lógicas tienen sus defectos y virtudes, y el de- 
bate sobre qué inferencias conviene adoptar involucra distintos tipos de valoración. En los casos que mencioné, asimismo, son los expertos (y no cualquier sujeto) los que desacuerdan como pares, y a diferencia de lo que sucede con los agentes no-formados (cuyas incoherencias son muchas veces fáciles de detectar), es muy difícil mostrar de forma conclusiva que los expertos que sostienen determinada lógica están equivocados. Por eso los expertos en lógica suelen ser receptivos con sus pares, incluso cuando sostienen posiciones distintas. La elección del grupo de expertos es en cierta medida arbitraria, aunque esa vaguedad podemos atribuirla al concepto de «experto» y no al desacuerdo lógico en particular.

\section{DESACUERDOS Y PRETENSIÓN DE UNIVERSALIDAD}

Otra cuestión problemática de mi propuesta está relacionada con la posibilidad de universalizar la votación de un grupo. A diferencia de lo que sucede en otros casos de agregación (supongamos, un grupo que decide qué pizza pedir), en la agregación lógica la votación que realiza un grupo pretende aplicarse a otros grupos.

Ciertamente existe allí una diferencia entre tipos de contextos para agregar creencias: no es lo mismo votar sobre pizzas que votar sobre lógicas. En la votación sobre lógicas parece existir una pretensión de alcanzar la verdad, y por eso el acuerdo entre un grupo reducido va a resultar insatisfactorio. Creo que hay dos maneras de responder esta cuestión. La primera es decir que un contexto ideal sería un desacuerdo lógico entre todos los expertos que estén en condición de paridad. Una agregación de ese tipo podría tener naturalmente una pretensión de universalidad. En segundo lugar, una agregación más realista podría reunir distintos agentes que se consideran pares. Si bien la respuesta alcanzada por ese grupo no podría universalizarse (en particular, si esos individuos consideran que hay otros pares epistémicos fuera del grupo), sí podría resumir los compromisos generales de ese grupo. Algo similar pasó con la lógica relevantista, donde un grupo de doce lógicos (Beall et al. 2012) escribieron un texto común expresando sus acuerdos respecto a los condicionales. Naturalmente, se refieren a los condicionales en el contexto relevantista, y no necesariamente pretenden aplicar ese análisis de los condicionales a otros enfoques (por ejemplo, a los estudios más lingüísticos o empíricos del condicional ordinario).

\section{¿IMPORTA la CANTIDAd DE AGENTES QUE ACEPTA CADA LógiCA?}

En la sección anterior, describí casos donde para resolver un problema hay diversas posiciones igualmente aceptables. Metodológicamente asumí que puede darse una agregación donde cada una de esas posibilidades cuente como un agente. Esto puede leerse de distintas maneras: por ejemplo, que cada lógica es aceptada por el mismo número de agentes. Pero eso no suele ocurrir. Otra manera (bastante más natural) de leerlo es que la cantidad de agentes que acepta cada lógica es irrelevante. Pero, ¿es esto así realmente?

En realidad, esta pregunta es más profunda: en casos de desacuerdo entre pares, existe un debate entre aquellos que sostienen que las «cantidades importan» (Lackey 2013) y los que sostienen que no (Kelly 2010). Los mecanismos de agregación que propongo son en realidad independientes de esa distinción. Asumí que las cantidades no importan para ejemplificar un caso donde la agregación da un resultado distinto a lo propuesto por los 
agentes individualmente. Sin embargo, si las cantidades importaran, y una abrumadora mayoría aceptara (supongamos) $K 3$, la lógica colectiva podría ser $K 3$. Los métodos de agregación pueden aplicarse del mismo modo: sólo hace falta rechazar la suposición metodológica de que cada opción razonable cuenta como un agente.

\section{¿ES LA SOLUCIÓN MAYORITARIA UNA MEJOR TEORÍA QUE LAS INDIVIDUALES?}

Otra pregunta que surge naturalmente es acerca de las teorías resultantes. Puede suceder que la teoría resultante de determinado método de agregación sea mucho peor que las teorías aceptadas individualmente (por ejemplo, que no satisfaga transitividad). Entonces, ¿por qué deberíamos usar estos métodos para resolver paradojas?

Este es un problema general de los métodos de agregación. La primera parte de este artículo puede verse como una respuesta negativa: la agregación no garantiza que se preserven algunas propiedades fundamentales de la consecuencia lógica, como la transitividad. En la segunda parte, sugiero que el método podría usarse de todos modos, sabiendo que algunas propiedades se preservan bajo el voto por mayoría (como la reflexividad y la monotonía) y otras no necesariamente. En un caso de paridad epistémica donde el consenso fuera difícil de conseguir, reglas como unanimidad y mayoría son formas razonables de encontrar una posición grupal. Este método tiene sus costos, obviamente.

La solución ideal sería que los individuos discutan y logren aceptar determinada lógica de forma unánime luego de la discusión. Pero aquí estamos hablando de casos en que esa discusión no llegó al destino pretendido, y se ha llegado a un escenario de paridad. Si el grupo aún tuviera que ponerse de acuerdo, las reglas de agregación son la forma más elegante de hacerlo. Si los agentes prefieren evitar el riesgo, pueden usar la regla de unanimidad; de este modo, la lógica grupal podrá ser más débil que las lógicas individuales, pero claramente va a satisfacer propiedades estructurales.

\section{¿CÓMO ESTABLECEMOS LAS RESTRICCIONES INICIALES?}

En la teoría presentada en el texto, asumo una serie de restricciones sobre las lógicas individuales: deben ser transitivas, reflexivas y monotónicas. Esto genera dos problemas.

En primer lugar, esta restricción admite lógicas disparatadas, como la lógica trivial, donde cualquier conjunto de premisas implica cualquier conclusión. Esto no me parece necesariamente problemático: las posturas trivialistas fueron defendidas por algunos filósofos (Kabay 2010). De todos modos, podrían agregarse otras restricciones, como que la lógica en cuestión no sea trivial. Ninguna de estas restricciones nuevas es particularmente fácil de determinar (¿cómo evitamos una lógica casi-trivial, donde todo esquema de inferencia sea válido salvo uno?), por eso he decidido no introducir principios de ese tipo. Una posibilidad compatible con lo que expuse en el artículo sería admitir solamente sublógicas de la lógica clásica: así podemos incluir a $K 3, L P, F D E$, etc. Quedarían afuera otras lógicas poco exploradas, como la lógica conexiva.

En segundo lugar, y más importante, la restricción prohíbe algunas lógicas que ciertos autores consideran aceptables, como las lógicas subestructurales. En el ámbito de la lógica, hay propuestas no-monotónicas (Makinson 2005), no-transitivas (Cobreros et al. 2013), no-contractivas (Zardini 2011) y hasta no-reflexivas (French 2016). En el marco que desarrollé, no se permite que los individuos acepten esas lógicas. 
Bien, la adopción de restricciones «tarskianas» (reflexividad, contracción, monotonía y sustitutividad) obedece a dos razones. La primera es sociológica: muchos filósofos consideran que esos son rasgos fundamentales de la lógica, y la gran mayoría de los lógicos no-clásicos también adoptan lógicas tarskianas. La segunda razón es metodológica: si no hubiera restricciones al empezar la agregación (más allá de la completitud y la consistencia), el voto sería idéntico a una decisión con una agenda de letras proposicionales. El fenómeno que describo, entonces, no tendría ninguna especificidad. Al establecer las restricciones de una lógica tarskiana, puedo mostrar un resultado interesante y no observado hasta ahora. Ahora bien, nada evita que los métodos de agregación puedan también aplicarse para desacuerdos entre lógicos no-estructurales. Si eso sucede, la obtención de lógicas grupales no-estructurales será absolutamente previsible.

\section{Conclusión}

En lógica suelen darse escenarios de paridad epistémica, en los que diversos agentes igualmente capaces defienden lógicas distintas y no hay argumentos concluyentes a favor o en contra de sus posiciones. Esto abre el lugar a la generación de consensos, donde por medio de mecanismos de agregación, los agentes que desacuerdan generan una perspectiva lógica colectiva. En este artículo, exploramos formalmente algunos de esos procesos de agregación.

Algunos mecanismos fueron desestimados. La regla de unanimidad genera lógicas colectivas demasiado débiles. La regla de premisas no es especialmente adecuada para la agregación de lógicas, porque no es clara la relación premisas-conclusión entre enunciados metateóricos sobre la validez de los razonamientos.

El mecanismo que estudiamos con más detalle fue la regla de la mayoría. En general, bajo condiciones bastante mínimas, la propiedad estructural de transitividad no se preserva utilizando esta regla. Este resultado puede servir para argumentar a favor de enfoques notransitivos, hoy por hoy bastante populares en filosofía de la lógica. Sin embargo, también hemos investigado condiciones bajo las cuales la sencilla regla de mayoría no amenaza nuestros principios estructurales. Hemos demostrado que esta regla puede dar un resultado estructural cuando tenemos perfiles organizados de ciertas maneras, como la alineación uniforme o una relación de inclusión «en $\mathrm{V}$ » entre las lógicas involucradas, donde dos lógicas distintas incluyen a una lógica más débil.

En resumen, hemos demostrado que muchas consideraciones sobre agregación de juicios pueden aplicarse a la agregación de lógicas. Dada la riqueza y variedad de los mecanismos de agregación de juicios, este proyecto tiene mucho más camino por recorrer.

\section{REFERENCIAS}

Beall, J.C, Ross Brady, John Michael Dunn, Allan Hazen, Edwin Mares, Robert Meyer, Graham Priest, Greg Restall, David Ripley y John Slaney. 2012. On the ternary relation and conditionality. Journal of Philosophical Logic 41, no. 3: 595-612. 
Briggs, Rachel, Fabrizio Cariani, Kenny Easwaran, and Branden Fitelson. 2014. Individual coherence and group coherence. En Essays in Collective Epistemology, editado por J. Lackey, 215-239. Oxford: Oxford University Press.

Carnielli, Walter, Marcelo Coniglio, Dov Gabbay, Paula Gouveia y Cristina Cernadas. 2008. Analysis and Synthesis of Logics: how to cut and paste reasoning systems. Dordrecht: Springer.

Cobreros, Pablo, Egré, Paul, Ripley, David, y Robert van Rooij. 2013. Reaching transparent truth. Mind 122, no. 488: 841-866.

Cook, Roy. 2005. What's wrong with tonk? Journal of Philosophical Logic 34, no. 2: 217-226.

Dietrich, Franz. 2007. A generalized model of judgement aggregation. Social Choice and Welfare 28, no. 4: 529-565.

Dietrich, Franz y Christian List. 2007. Arrow's theorem in judgment aggregation. Social Choice and Welfare 29, no. 1:19-33.

Field, Hartry. 1998. Epistemological non-factualism and the aprioricity of logic. Philosophical Studies 92, no. 1-2: 1-24.

French, Rohan. 2016. Structural reflexivity and the paradoxes of self-reference. Ergo 3, no. 5 : 113-131.

Gaertner, Wulf. 2009. A Primer in Social Choice Theory. Oxford: Oxford University Press.

Halbach, Volker. 2011. Axiomatic theories of truth. Oxford: Oxford University Press.

Horsten, Leon y Volker Halbach. 2006. Axiomatizing Kripke's Theory of Truth. Journal of Symbolic Logic 71, no. 2: 677-712.

Kabay, Paul. 2010. On the plenitude of truth. Saarbrücken: Lambert Academic Publisher.

Kelly, Thomas. 2005. The epistemic significance of disagreement. En Oxford Studies in Epistemology 1, editado por Hawthorne, J. y T. Gendler, 167-196. Oxford: Oxford University Press.

Kelly, Thomas. 2010. Peer disagreement and higher order evidence. En Social Epistemology: Essential Readings, editado por A. Goldman y D. Whitcomb, 183-217. Oxford: Oxford University Press.

Kripke, Saul. 1975. Outline of a theory of truth. Journal of Philosophy 72, no. 19: 690-716.

Lackey, Jennifer. 2013. Disagreement and Belief Dependence: Why Numbers Matter. En The Epistemology of Disagreement: New Essays, editado por D. Christensen y J. Lackey, 243-268. Oxford: Oxford University Press.

List, Christian y Phillip Pettit. 2002. Aggregating sets of Judgments: An Impossibility Result. Economics and Philosophy 18, no. 1: 89-110.

List, Christian y Phillip Pettit. 2004. Aggregating sets of judgements: Two impossibility results compared. Synthese 140, no. 1-2: 207-235.

List, Christian. 2012. Judgment Aggregation: An introductory review. Synthese 187, no. 1: 179-207.

List, Christian. 2015. When to defer to supermajority testimony. En Essays on Collective Epistemology. Editado por J. Lackey, 240-250. Oxford: Oxford University Press.

Makinson, David. 1965. The Preface Paradox. Analysis 25, no. 6: 205-207.

Makinson, David. 2005. Bridges from classical to non-monotonic logic. Londres: College Publications.

Miller, Michael y Daniel Osherson. 2009. Methods for distance-based judgement aggregation. Social Choice and Welfare 32, no. 4: 575-601.

Pettit, Phillip. 2001. Deliberative democracy and the discursive dilemma. Philosophical Issues 11, no. 1:268299.

Pigozzi, Gabriella. 2006. Belief merging and the discursive dilemma. Synthese 152 , no. 2: 285-298.

Pigozzi, Gabriella. 2014. A primer on Judgment Aggregation. San Rafael, California: Morgan \& Claypool Publishers.

Priest, Graham. 2006. In contradiction. Oxford: Oxford University Press.

Rahwan, Iyad y Fernando Tohmé. 2010. Collective argument evaluation as judgement aggregation, Proceedings of $9^{\text {th }}$ AAMAS.

Weber, Zach, Patrick Girard y Guillermo Badia. 2015. What is an inconsistent truth table, Australasian Journal of Philosophy, en prensa. 
Williamson, Timothy. 2013. Semantic paradoxes and abductive methodology. En The Revenge of the Liar, editado por Armour-Garb, B. Oxford: Oxford University Press, en prensa.

Zardini, Elia. 2011. Truth without contra(di)ction. Review of Symbolic Logic 4, no. 4: 498-535.

Diego TAjer es doctor en Filosofía por la Universidad de Buenos Aires y ayudante de Lógica en la misma institución. Sus principales áreas de investigación son las paradojas semánticas, la filosofía de la lógica y la epistemología formal. Actualmente es becario postdoctoral del Consejo Nacional de Investigaciones Científicas y Técnicas (CONICET). Sus artículos han sido publicados en Journal of Philosophical Logic y próximamente en Synthese y Logique et Analyse.

Dirección: Universidad de Buenos Aires / Instituto de Investigaciones Filosóficas, Sociedad Argentina de Análisis Filosófico (SADAF), Consejo Nacional de Investigaciones Científicas y Técnicas (CONICET), Bulnes 642, 1176, Buenos Aires, Argentina. E-mail: diegotajer@gmail.com 